\title{
Engineering Project Review Method Based on Matter-Element and Hierarchy Model
}

\author{
Haifeng $\mathrm{LI}^{1, \mathrm{a}}$ \\ 1Dalian Jiaotong University, No.794 Huanghe Road, Shahekou District, Dalian, 116028, China \\ ahaifengli@dlut.edu.cn
}

Keywords: project review method; peer communication review; matter-element and hierarchy model; engineering project review

Abstract. Project review is always the key link of the engineering project management. Project review is a complex work, which involves many factors. The final review result is always influenced directly by the definition of various evaluation indexes and the corresponding weight. Mainly study the judgment of the experts' ability and the establishment of the project evaluation index system in the peer communication review, analyze deeply the engineering project review work, build a evaluation index system for the engineering project, and put forward a comprehensive evaluation method based on the matter-element and hierarchy model in the engineering project, at last, apply it in the actual project which proves the practicability of the paper's theory.

\section{Introduction}

The impartiality of the engineering project review is very important, which most applicants pay close attention to. Nowadays, peer communication review [1] is commonly used in the review work, which means experts of the same field review the project back to back. Peer communication review can take full advantage of scientists who are in the scientific community to realize the reasonable allocation of scientific and technological resources. It also contributes to the democratization of the scientific decision-making, and puts an end to the purely administrative decision-making.

This article attempts to establish the evaluation index system of the engineering project, give the knowledge set representation of engineering project knowledge, put forward engineering project evaluation method based on the matter-element and hierarchy model [2], and at last validates it through an actual project.

\section{Project evaluation index system}

Table 1. Project evaluation index system

\begin{tabular}{ll}
\hline Comprehensive index & Single index \\
\hline Research goal $\left(b_{1}\right)$ & Scientific level $\left(c_{1}\right)$ \\
& Expected results $\left(c_{2}\right)$ \\
& Application prospect $\left(c_{3}\right)$ \\
Research content $\left(b_{2}\right)$ & Implementation content $\left(c_{4}\right)$ \\
& Technical route $\left(c_{5}\right)$ \\
& Schedule $\left(c_{6}\right)$ \\
Research ability $\left(b_{3}\right)$ & Budget $\left(c_{7}\right)$ \\
& Pre-implementation work $\left(c_{8}\right)$ \\
& Implementation ability $\left(c_{9}\right)$ \\
& Implementation conditions $\left(c_{10}\right)$ \\
\hline
\end{tabular}

The evaluation of engineering project involves many factors, such as the rationality of project contents, the ability of the executors, and the application prospect of the engineering project, which all need carrying on the comprehensive evaluation. In this paper, we define a comprehensive evaluation index system for the project to be reviewed. The system contains three comprehensive indexes and ten single indexes, as shown in table 1. 


\section{Matter-element representation of project knowledge}

The project as a knowledge carrier, it contains the knowledge is called project knowledge, according to matter-element, project knowledge can be represented as

$$
K S_{P}=\left(N_{P}, C, V\right)=\left[\begin{array}{ccc}
N P & c_{1} & v_{1} \\
& c_{2} & v_{2} \\
& c_{3} & v_{3} \\
& \cdots & \cdots \\
& c_{n} & v_{n}
\end{array}\right]
$$

where $\mathrm{N}_{P}$ is project, $\mathrm{C}$ is the project's feature set $\left\{\mathrm{c}_{1}, \mathrm{c}_{2}, \ldots, \mathrm{c}_{\mathrm{n}}\right\}, \mathrm{V}$ is quantum set of $\mathrm{N}$ about $\mathrm{C}\left\{\mathrm{v}_{1}\right.$, $\left.\mathrm{v}_{2}, \ldots, \mathrm{v}_{\mathrm{n}}\right\}$.

\section{Classical domain and section domain}

Classical domain matter-element is defined as

$$
R_{j}=\left(N_{j}, C, X_{j}\right)=\left[\begin{array}{ccc}
N_{j} & c_{1} & X_{j 1} \\
& c_{2} & X_{j 2} \\
& \cdots & \ldots \\
& c_{n} & X_{j n}
\end{array}\right]=\left[\begin{array}{ccc}
N_{j} & c_{1} & <a_{j 1}, b_{j 1}> \\
& c_{2} & <a_{j 2}, b_{j 2}> \\
& \ldots & \ldots \\
& c_{n} & <a_{j n}, b_{j n}>
\end{array}\right],
$$

where $\mathrm{N}_{\mathrm{j}}(\mathrm{j}=1,2, \ldots, m)$ is the $\mathrm{j}$-grade status of the review; $\mathrm{c}_{\mathrm{i}}(\mathrm{i}=1,2, \ldots, n)$ is the corresponding characteristic for a grade status of the review; interval $X_{j i}=\left\langle a_{j i}, b_{j i}>\right.$ is the range of the value $c_{i}$ for $N_{j}$, namely, the data range of the corresponding index of the each grade of the review status-classical domain.

Section domain matter-element is defined as

$$
R_{p}=\left(N_{p}, C, X_{p}\right)=\left[\begin{array}{ccc}
N_{p} & c_{1} & X_{p 1} \\
& c_{2} & X_{p 2} \\
& \ldots & \ldots \\
& c_{n} & X_{p n}
\end{array}\right]=\left[\begin{array}{ccc}
N_{p} & c_{1} & <a_{p 1}, b_{p 1}> \\
& c_{2} & <a_{p 2}, b_{p 2}> \\
& \ldots & \ldots \\
& c_{n} & <a_{p n}, b_{p n}>
\end{array}\right],
$$

where $\mathrm{N}_{\mathrm{p}}$ is the whole evaluation grade; $\mathrm{c}_{\mathrm{i}}(\mathrm{i}=1,2, \ldots, \mathrm{n})$ is a characteristic of the whole grade; interval $\mathrm{X}_{\mathrm{pi}}=\left\langle\mathrm{a}_{\mathrm{pi}}, \mathrm{b}_{\mathrm{pi}}>\right.$ is the range of the value $\mathrm{c}_{\mathrm{i}}$ for $\mathrm{N}_{\mathrm{p}}$, namely, the data range of the index of the whole review grade----section domain. Obviously, there is $\mathrm{X}_{\mathrm{j}} \subset \mathrm{X}_{\mathrm{p}}$.

\section{Correlation function}

Correlation function [3] describes the degree of the required level of the matter-element, giving the matter-element value $\mathrm{x}$ mapping to the real axis in the matter-element analysis evaluation. The distance between node $x_{i}$ and limited reality interval $X=<a, b>$ is defined as

$$
\rho\left(\mathrm{x}_{\mathrm{i}}, \mathrm{X}\right)=\left|\mathrm{X}_{\mathrm{i}}-\frac{\mathrm{a}+\mathrm{b}}{2}\right|-\frac{1}{2}(b-a)= \begin{cases}a-x_{\mathrm{i}} & x_{\mathrm{i}} \leq \frac{a+b}{2} \\ x_{\mathrm{i}}-b & x_{\mathrm{i}}>\frac{a+b}{2}\end{cases}
$$

where the distance between node $x_{i}$ and interval $X$ can be negative value, the difference of the negative value determines correspondingly the difference of the position $\mathrm{x}_{\mathrm{i}}$ in the interval $\mathrm{X}$. 
We can get the distance between the node $\mathrm{x}_{\mathrm{i}}$ and the interval $\mathrm{X}_{\mathrm{ji}}$ of classical domain, as well as the distance between the node $\mathrm{xi}$ and the interval $\mathrm{X}_{\mathrm{pi}}$ of section domain as

$$
\begin{aligned}
& \rho\left(\mathrm{x}_{\mathrm{i}}, \mathrm{X}_{\mathrm{ji}}\right)=\left|\mathrm{xi}_{\mathrm{i}}-\frac{a_{\mathrm{ji}}+\mathrm{b}_{\mathrm{ji}}}{2}\right|-\frac{1}{2}\left(b_{\mathrm{ji}}-a_{\mathrm{ji}}\right)= \begin{cases}a_{\mathrm{ji}}-x_{\mathrm{i}} & x_{\mathrm{i}} \leq \frac{a_{\mathrm{ji}}+b_{\mathrm{ji}}}{2} \\
x_{\mathrm{i}}-b_{\mathrm{ji}} & x_{\mathrm{i}}>\frac{a_{\mathrm{ji}}+b_{\mathrm{ji}}}{2}\end{cases} \\
& \rho\left(\mathrm{x}_{\mathrm{i}}, \mathrm{X}_{\mathrm{pi}}\right)=\left|\mathrm{x}_{\mathrm{i}}-\frac{\mathrm{a}_{\mathrm{pi}}+\mathrm{b}_{\mathrm{pi}}}{2}\right|-\frac{1}{2}\left(b_{\mathrm{pi}}-a_{\mathrm{pi}}\right)= \begin{cases}a_{\mathrm{pi}}-x_{\mathrm{i}} & x_{\mathrm{i}} \leq \frac{a_{\mathrm{pi}}+b_{\mathrm{pi}}}{2} \\
x_{\mathrm{i}}-b_{\mathrm{pi}} & x_{\mathrm{i}}>\frac{a_{\mathrm{pi}}+b_{\mathrm{pi}}}{2}\end{cases}
\end{aligned}
$$

Then the computation formula of the correlation function $\mathrm{K}(\mathrm{x})$ is defined as

$$
K_{j}\left(x_{i}\right)=\left\{\begin{array}{cc}
\frac{\rho\left(x_{i}, X_{j i}\right)}{\rho\left(x_{i}, X_{p i}\right)-\rho\left(x_{i}, X_{j i}\right)} & x_{i} \notin X_{j i} \\
-\frac{\rho\left(x_{i}, X_{j i}\right)}{\left|X_{j i}\right|} & x_{i} \in X_{j i}
\end{array}\right.
$$

Correlation function describes the degree of the required level of the matter-element, giving matter-element the value $\mathrm{x}_{0}$ mapping to the real axis in the matter-element analysis evaluation. Actually, it describes the degree of ownership of the each index of the project to be reviewed about each review grade $\mathrm{j}$, which makes mathematics "belong" and "not belong" qualitative description the extended to quantitative description.

\section{Project matter-element to be evaluated}

If there are $\mathrm{n}$ single indexes in the comprehensive index $b_{k}$, namely, $c_{i}(i=1,2, \ldots, n)$, and the corresponding single index matter-element to be reviewed is $R_{c}$, we can define the single index matter-element as

$$
R_{c}=\left(N_{o}, C, \mathrm{~V}_{\mathrm{c}}\right)=\left[\begin{array}{ccc}
N_{o} & c_{1} & \mathrm{~V}_{\mathrm{c} 1} \\
& c_{2} & \mathrm{~V}_{\mathrm{c} 2} \\
& \ldots & \ldots \\
& c_{n} & \mathrm{~V}_{\mathrm{cn}}
\end{array}\right],
$$

where $\mathrm{N}_{\mathrm{o}}$ is the project to be reviewed; $\mathrm{C}$ is the whole characteristics of the comprehensive index $b_{k}(k=1,2, \ldots, m)$ of $N_{o}$, namely, all the single indexes $c_{i}(i=1,2, \ldots, n)$ of the comprehensive index $b_{k}$ of $\mathrm{N}_{\mathrm{o}} ; \mathrm{v}_{\mathrm{ci}}$ is the value of the single index $\mathrm{c}_{\mathrm{i}}$.

If there are $\mathrm{m}$ experts to review the project $\mathrm{N}_{\mathrm{o}}, \mathrm{v}_{\mathrm{ci}}$ being the comprehensive value of the single index $\mathrm{c}_{\mathrm{i}}$ scored by the expert group, $\mathrm{v}_{\mathrm{ci}}$ is defined as

$$
v_{c i}=\sum_{k=1}^{m}\left(v_{\mathrm{i} k} \times z k\right)
$$

where $v_{i k}$ is the value of single index $c_{i}$ scored by the expert $k, z_{k}$ is the weight of expert $k$ in the expert group.

If there are $m$ comprehensive indexes in the project, namely, $b_{i}(i=1,2, \ldots, m)$, the comprehensive index matter-element $R_{b}$ to be evaluated is defined as 


$$
R_{b}=\left(N_{o}, B, \mathrm{~V}_{\mathrm{b}}\right)=\left[\begin{array}{ccc}
N_{o} & b_{1} & \mathrm{Vb} 1 \\
& b_{2} & \mathrm{Vb} 2 \\
& \ldots & \ldots \\
& b_{m} & \mathrm{Vb} m
\end{array}\right],
$$

where $\mathrm{N}_{\mathrm{o}}$ is the project to be reviewed, $\mathrm{B}$ is the characteristics of $\mathrm{N}_{\mathrm{o}}$, namely, the comprehensive indexes of $\mathrm{N}_{\mathrm{o}} ; \mathrm{v}_{\mathrm{bk}}$ is the value of the comprehensive index $\mathrm{b}_{\mathrm{k}}(\mathrm{k}=1,2, \ldots, \mathrm{m})$. If the comprehensive index $b_{k}$ is composed of single index $c_{i}(i=1,2, \ldots, l), v_{b k}$ is defined as

$$
v_{b k}=\sqrt[l]{\prod_{i=1}^{l} v_{c i}},
$$

Define the classical domain and section domain of the comprehensive index matter-element, according to the formula (2), (3). Then calculate the correlation of each comprehensive index for each grade of the review status.

After introducing the weight of comprehensive index, define the correlation of $\mathrm{N}_{\mathrm{o}}$ for $\mathrm{j}$ grade of the review status as

$$
K_{j}\left(N_{o}\right)=\sum_{i=1}^{n} \omega_{i} K_{j}\left(b_{i}\right)
$$

where $\mathrm{n}$ is the number of the comprehensive indexes, $\mathrm{b}_{\mathrm{i}}(\mathrm{i}=1,2, \ldots, \mathrm{n})$ is the ith comprehensive index, $\omega_{i}$ is the weight of the comprehensive index $b_{i}$.

If $\max \left(\mathrm{K}_{\mathrm{j}}\left(\mathrm{N}_{\mathrm{o}}\right)\right)=\mathrm{K}_{\mathrm{jo}}$, we can conclude that the matter-element to be reviewed belongs to $\mathrm{j}_{\mathrm{o}}$ grade.

If $\mathrm{K}_{\mathrm{jo}}\left(\mathrm{N}_{\mathrm{o}}\right)>1$, it means that the matter-element to be evaluated exceeds the upper limit of the standard grade. The bigger its value is, the greater the potential for development is.

\section{Conclusion}

The review work is a complex work which involves many aspects of factors in the engineering project. The final evaluation result is influenced directly according to the definition of various evaluation indexes and the corresponding weight. This paper is based on the matter-element analysis, considering fully the factors of the project itself and the review expert, establishing a comprehensive evaluation index system and comprehensive evaluation algorithm with fairness, operability.

\section{Acknowledgements}

This research was financially supported by the National Natural Science Foundation of China (Grant NO. 71301016), the Social Science Foundation of Liaoning Province (Grant NO. L15BGL002) and the Outstanding Talent Support Program of Liaoning Province (Grant NO. WJQ2015006).

\section{References}

[1]Harjumaa L, Tervonen I, Huttunen A. Peer review in real life-motivators and demotivators [C]. Quality Software (QSIC2005), 2005, 9:29-36.

[2]Haifeng Li, Yanzhong Dang. Comprehensive evaluation method research based on matter-element analysis in fund project review[C]. 2nd International Asia Conference on Informatics in Control Automation and Robotics, CAR 2010, Wuhan, China, 2010:496-499.

[3]Xiuling Sun. Matter element extension evaluating model based on synthesis weights and approach degree and its application [C]. USA: 2nd International Con-ference on Bioinformatics and Biomedical Engineering, 2008. 